\title{
Maser science at Tidbinbilla
}

\author{
Shinji Horiuchi ${ }^{1}$ and Jim Lovell ${ }^{2} \dagger$ \\ ${ }^{1}$ Canberra Deep Space Communication Complex, Raytheon Australia, PO Box 1035, \\ Tuggeranong, ACT 2901, Australia \\ email: shoriuchi@cdscc.nasa.gov \\ ${ }^{2}$ CSIRO Australia Telescope National Facility and CSIRO Industrial Physics, NSW, Australia \\ email: jim.lovell@utas.edu.au
}

\begin{abstract}
The $70 \mathrm{~m}$ antenna at Canberra Deep Space Communication Complex (Tidbinbilla) is the largest and most sensitive dish in the southern hemisphere, working at 1,3,13 and $18 \mathrm{~cm}$, and as such is in high demand from astronomers both in Australia and overseas. In this paper we present the current status of the single dish spectroscopy system and highlight some recent results in maser science.
\end{abstract}

\section{1. $12 \mathrm{~mm}$ system and spectrometer}

The Canberra Deep Space Communications Complex (CDSCC) at Tidbinbilla is part of NASA's Deep Space Network (DSN) and is home to the largest and most sensitive single dish in the southern hemisphere. This 70-m antenna is primarily used for spacecraft tracking but, as part of the Host Country agreement with NASA, a fraction of time on the antenna is allocated for independent scientific activities sponsored by the Australian Government. This time allocation, typically 300 hours per year, is divided into single-dish and VLBI observing. Access to this time is obtained via proposal through the ATNF and is open to anyone.

Of particular interest is the $12 \mathrm{~mm}(18-26 \mathrm{GHz})$ system. With a SEFD of $60 \mathrm{Jy}$ it is 10 times more sensitive than the current Parkes system and when the Parkes $12 \mathrm{~mm}$ receiver is upgraded will still be 3.5 times more sensitive. The $12 \mathrm{~mm}$ system at Tidbinbilla currently operates with an on-axis feed and provides dual-polarisation output with an instantaneous available bandwidth of $600 \mathrm{MHz}$. An upgrade is currently underway to provide a simultaneous off-axis beam to allow simultaneous on- and off-source spectra to be taken, effectively halving the required observing time for pointed observations.

Currently Tidbinbilla is equipped with a Parkes Multibeam correlator block which allows two IFs to be correlated at a maximum bandwidth of $64 \mathrm{MHz}$. For more information see http://www.atnf.csiro.au/observers/tidbinbilla. It is hoped that a new spectrometer will soon be obtained to provide sufficient bandwidth and channelisation to handle all four IFs from the upgraded front-end.

\section{Galactic masers}

Ellingsen et al. (2004) searched for $19.9 \mathrm{GHz}$ methanol masers in star-forming regions using the $70 \mathrm{~m}$ telescope and found six new sources. The results indicate a strong correlation between $19.9 \mathrm{GHz}$ methanol masers and the Class II methanol masers associated with $6035 \mathrm{MHz} \mathrm{OH}$ masers.

$\dagger$ Present address: Department of Maths and Physics, University of Tasmania, Private Bag 21, Hobart, Tasmania 7001, Australia 
Valdettaro et al. (2007) performed a high sensitivity survey of water masers in bright rimmed clouds compressed by ionization fronts from nearly massive stars. They found masers from seven bright rimmed interstellar clouds out of 44 such regions. The low detection rate suggests that low-mass star formation is the most natural outcome of such external compression.

Deacon et al. (2007) observed a sample of 85 post-Asymptotic Giant Branch (postAGB) candidate stars, selected on the basis of their $\mathrm{OH} 1612 \mathrm{MHz}$ and far-infrared properties. New detections of water masers were identified for 21 stars. The source b292 is a young post-AGB star that is highly likely to be a 'water fountain' source with highly collimated, high velocity (up to $210 \mathrm{~km} / \mathrm{s}$ ) water masers. (See Chapman et al. in these proceedings.)

\section{Extragalactic masers}

Greenhill et al. (2003a) discovered water maser emission in seven AGN using a custombuilt 4096 channel spectrometer with a bandwidth of $5300 \mathrm{~km} / \mathrm{s}$. A continuation of the search was made, resulting in new detection in eight more AGN (Kondratko et al. 2006). Greenhill et al. (2003a) also discovered high velocity components in the Seyfert nucleus of the Circinus galaxy.

Tarchi et al. (2007, in preparation) have been searching for water maser emission toward a sample comprised of all galaxies with Dec. $<30$ deg with IRAS 100 micron fluxes $>50 \mathrm{Jy}$ in order to confirm the relation between FIR flux and water maser detection rate found in the northern hemisphere (Henkel et al. 2005). While this survey is still on going, so far they have detected water masers in one of the sources in the sample.

Greenhill et al. (2003b) presented water maser VLBI maps of the the Circinus galaxy obtained by the Australian Long Baseline Array (LBA) in which the Tidbinbilla 70m played a key role as the most sensitive antenna in the array. The outflowing masers are irregularly distributed above and below a warped, edge-on accretion disk (maximum rotation speed $260 \mathrm{~km} / \mathrm{s}$ ), with relative outflow velocities up to $\pm 160 \mathrm{~km} / \mathrm{s}$, projected along the line of sight.

Greenhill et al. (1997) have discovered that the water maser lines in the Circinus galaxy vary on time scales as short as a few minutes. Follow up observations were made at Tidbinbilla by McCallum et al. (2005) and the same phenomena were confirmed. McCallum et al. (2005) showed that this rapid variability can be explained by interstellar scintillation, similar to that seen in a significant number of flat-spectrum AGN.

\section{References}

Deacon, R. M., Chapman J. M., Green, A. J., Sevenster, M.N 2007, ApJ 658, 1096

Ellingsen, S. P., Cragg, D. M., Lovell, J. E. J., Sobolev, A. M., Ramsdale, P. D., Godfery, P. D. 2004, MNRAS 354, 401

Greenhill, L. J., Ellingsen, S. P., Norris, R. P., Gough, R. G., Sinclair, M. W., Moran, J. M., Mushotzky, R. 1997, ApJ 464, L103

Greenhill, L. J., Kondratko, J. E., Lovell, J. E. J., Kuiper, T. B. H., Moran, J. M., Jauncey, D. L., Baines, G. P. 2003, ApJ 582, L11

Greenhill, L. J., Booth, R. S., Ellingsen, S. P., Herrnstein, J. R., Jauncey, D. L., McCulloch, P. M., Moran, J. M., Norris, R. P., Reynolds, J. E., Tzioums, A. K. 2003, ApJ 590, L162

Kondratko, P. T., Greenhill, L. J., Moran, J. M., Lovell, J. E. J., Kuiper, T. B. H., Jauncey, D. L., Cameron, L. B., Gómez, J. F., García-Miró, C., Moll, E., de Gregorio-Monsalvo, I., Jiménez-Bailón, E. 2006, ApJ 638,100

McCallum, J. N., Ellingsen, S. P., Jauncey, D. L., Lovell, J. E. J., Greenhill, L. J. 2005, AJ 129, 1231

Valdettaro, R., Chapman, J. M., Lovell, J. E. J., Palla, F. 2007, A $\& A$ 466, 247 

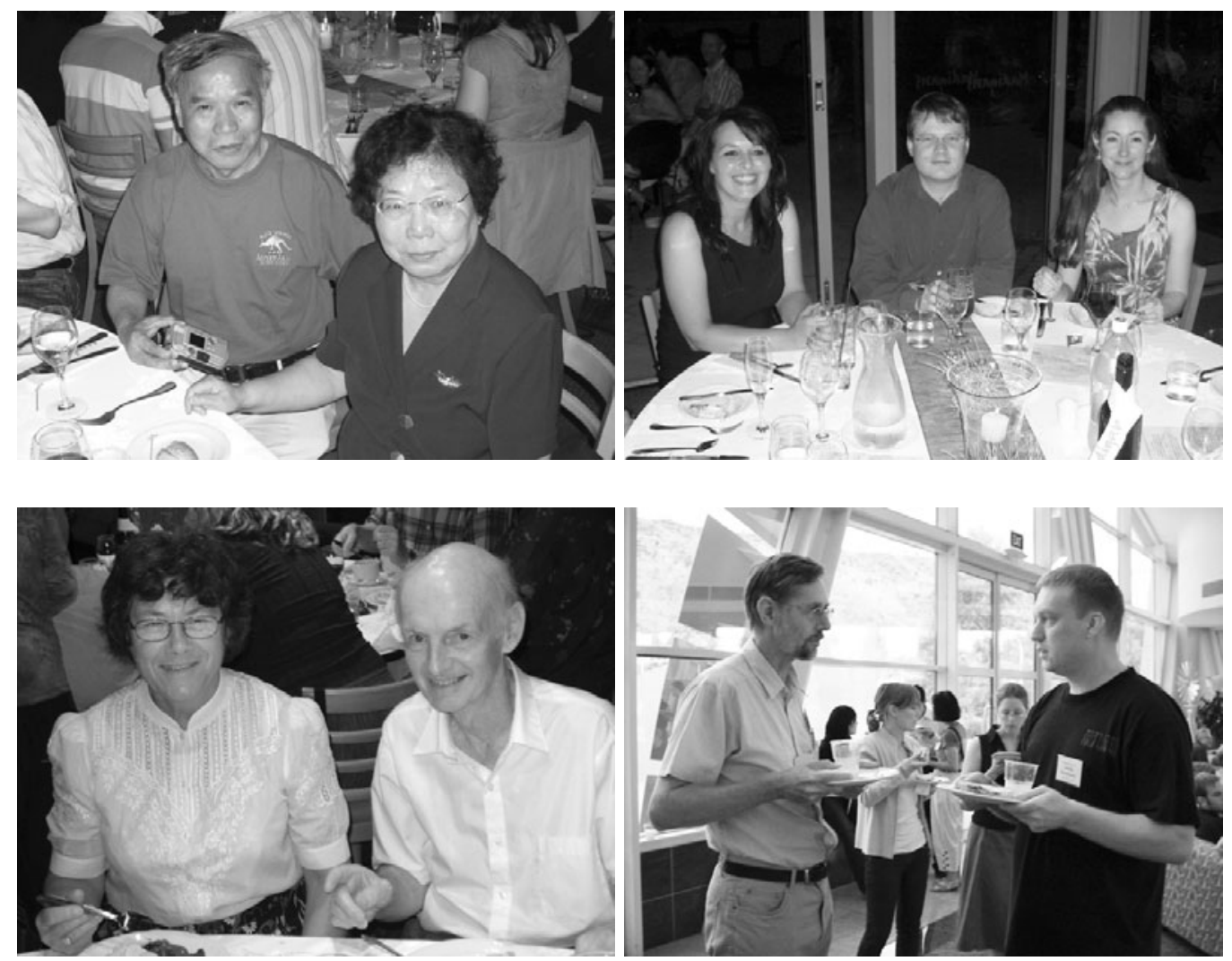

Photos (clockwise from top left): James Moran, James Moran, Andrej Sobolev, Miller Goss

Top row: Professor Xingwu Zheng with Associate Professor Zhaofen Ling (left) Vicki Drazenovic, Todd Hunter and Crystal Brogan (right)

Bottom row: James and Sheena Caswell (left),

Michael Gaylard and Leszek Blaszkiewicz (right) 